\title{
Anisotropic Stimulated Emission from Aligned CdSe/CdS Dot-in-Rods
}

\author{
Yuan Gao, ${ }^{a, b}$ Van Duong Ta, ${ }^{a}$ Xin Zhao, ${ }^{a, b}$ Yue Wang, ${ }^{a}$ Rui Chen, ${ }^{a, b}$ Yongbiao Zhao, ${ }^{b}$ Cuong Dang, ${ }^{b}$ Xiaowei \\ Sun $^{b, c}$, Handong Sun, ${ }^{a, b,+}$ and Hilmi Volkan Demir ${ }^{a, b, c,+}$ \\ ${ }^{a}$ Division of Physics and Applied Physics, School of Physical and Mathematical Sciences, Nanyang \\ Technological University, Singapore 637371 \\ ${ }^{b}$ Luminous! Center of Excellence for Semiconductor Lighting and Displays, School of Electrical and Electronic \\ Engineering, Nanyang Technological University, Singapore 639785 \\ ${ }^{c}$ Department of Electrical and Electronics Engineering, Department of Physics, UNAM-Institute of Materials \\ Science and Nanotechnology, Bilkent University, Ankara 06800, Turkey \\ Email: hdsun@ntu.edu.sg;hvdemir@ntu.edu.sg
}

\begin{abstract}
Anisotropic optical properties of $\mathrm{CdSe} / \mathrm{CdS}$ dot-in-rods loaded in a capillary tube are demonstrated, suggesting nanorods' alignment with a microfluidic approach. Polarized emissions from photoluminescence and whispering gallery mode lasing show promising applications for lighting and displays.

Keywords - polarization; WGM; semiconductor nanocrystal; nanorod.

Semiconductor nanocrystals demonstrated high photoluminescence (PL) efficiency with wide emission wavelength tunability by simply tailoring the size, structure or chemical composition.[1] Given their larger absorption cross section, narrow emission spectra, and better photostability compared to organic dyes, semiconductor nanocrystals are widely used as fluorescence materials in bio-labeling, LED down-converting, or direct LED applications. Their atomic like states improve the temperature insensitivity, promising a very attractive material for lasing applications.[2] Elongate semiconductor nanocrystals, such as nanorods, exhibit anisotropic optical properties due to asymmetry of intrinsic wurtzite lattice structure [3] and extrinsic shapes. The CdSe seeded $\mathrm{CdS}$ rods, CdSe/CdS dot-in-rods (DRs) have been synthesized recently and displayed high PL efficiency, tunability, large absorption, and large Stokes shift. [4] Due to the quasi-type-II band alignment, Auger recombination in CdSe/CdS DRs is suppressed and gain lifetime increases remarkably.[5] Furthermore, colloidal nanocrystal laser enabled by single exciton gain mechanism was demonstrated [6] with this material. As nature of asymmetric nanocrystals, the single DR also shows anisotropic photoluminescence [7] and absorption [8]. But the random distribution of DRs in previous laser experiment [6] would not make use of these anisotropic optical properties. Many efforts have been paid to develop nanorod superlattices to exploit anisotropic optical properties in a macroscopic scale. [7, 9, 10] Here, we developed a method to align DRs
\end{abstract}

macroscopically to make a new anisotropic gain medium with desired properties of nanocrystals.[11] By drying DRs solution which is loaded in a capillary, the microfluid induces shear flow and aligns nanorods along the flow direction.[12] Multilayers of aligned DRs are deposited on the side wall of the capillary and form an anisotropic gain medium incorporated within optical microring cavity.[11]

We adopt two methods to examine the optical anisotropy of aligned DRs in a capillary (Fig. 1).[11] Firstly, the DRs loaded in a capillary are excited by varying linear polarized light, and a detector collects the PL spectra (Fig. 1a) to show the anisotropic absorption. Secondly, the same sample is illuminated by circular polarized light, and by tuning the polarizer in front of PL collection, polarization dependent PL is recorded (Fig. 1b). Fig. 1c shows PL intensities as functions of excitation and emission polarization angles. The results show both anisotropic absorption and emission of DRs in a capillary with a peak at $\theta=$ 90 degree (parallel to the capillary axis) and minimized at $\theta=0$ degree (normal to the capillary axis). The experiment data can be well fitted with a function: $I=E^{2} \sin ^{2} \theta+C$, where $\mathrm{E}$ is electric field, $\mathrm{C}$ is background constant. For a single nanorod of wurtzite lattice structure, the transitions along the c-axis have larger possibility, which leads to higher absorption and emission. Therefore, the polarization spectroscopy results suggest the DRs in capillary tube align parallel to the tube, with caxis along the axis of the capillary. Meanwhile, control experiments with spherical quantum dots were conducted to rule out the influence of cavity behavior and polarization dependent response of the detector. The degree of optical anisotropy in emission and absorption of DRs in the capillary, characterized by polarization difference ratio $\gamma=\frac{I_{\|}-I_{\perp}}{I_{\|}+I_{\perp}}$, are 0.252 and 0.296 respectively. 


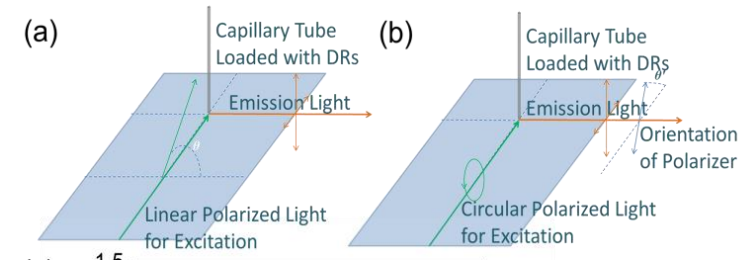

(c) $\simeq 1.5$. Circular Polarized Light Excitation

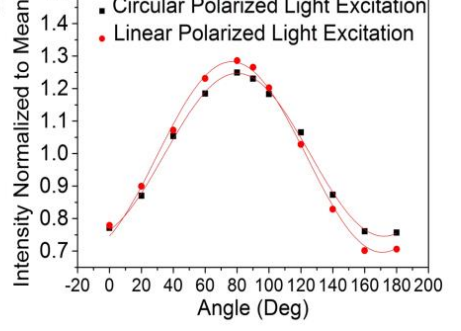

Figure 1. Two schemes for examining polarization dependent absorption and emission. (a) Linear polarized light excitation (anisotropic absorption): tuning polarization orientation of excitation and monitoring the PL intensity. (b) Circular polarized light excitation (anisotropic emission): tuning a polarizer in front of detector and monitor the PL intensity. (c) Normalized PL intensities as functions of polarization angle for two different schemes.

For stimulated emission from the DRs in capillary, more DRs are deposited onto the inner wall of capillary to achieve enough gain.[11] The sample was pumped with a pulsed laser (1 ns pulse width and $532 \mathrm{~nm}$ wavelength). After passing through a quarter wave plate, the circular polarized laser beam excites the sample as in Fig. 1 (b). When the excitation is below threshold of $12 \mathrm{~mJ}$, the PL of the structure is observed as normal DRs' spontaneous emission (FWHM of $34.7 \mathrm{~nm}$ ). With above threshold excitation, the stimulated emission within the micro-ring cavity clearly shows narrow whispering gallery modes with equal spacing and stable positions (Fig. 2a). The emission intensities show a well-defined threshold behaviour for both polarizations. The power transfer function was fitted very well with "soft threshold" approximation (Fig. 2b).[13] Another observation is the polarization difference ratio built up with increasing pumping power as shown in Fig. 2 (b). The result suggests the competition between the preferred polarization of align DRs and the polarization of dominant cavity modes.

In conclusion, we show alignment of nanorods in a large scale.[11] The degree of anisotropic absorption and emission from DRs in capillary was measured. In addition, the anisotropic stimulated emission with well-defined lasing modes was demonstrated. The results can be utilized for lighting and display applications.

\section{ACKNOWLEDGMENT}

This work is supported by the Singapore Ministry of Education through the Competitive Research Programme (CRP) under Projects No. NRF-CRP62010-02 and NRF-CRP5-2009-04).

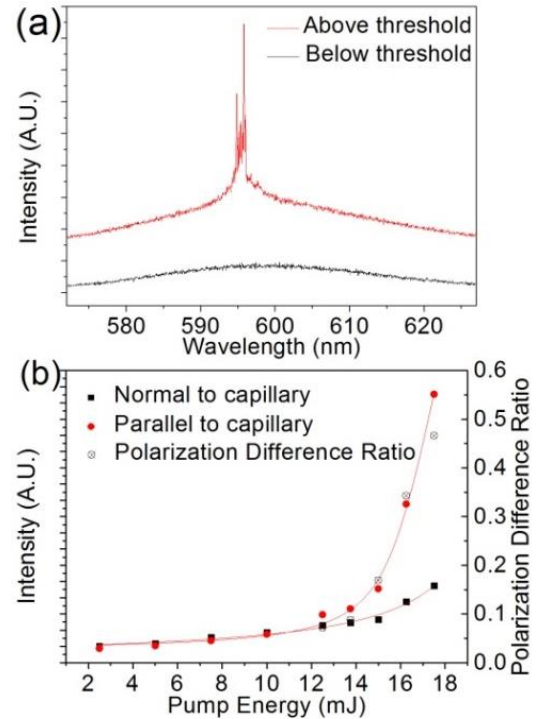

Figure 2. Stimulated emission from DRs in capillary. (a) Spectra of DRs' emission with excitation below and above threshold. (b) Power transfer function for emission from orientation parallel $\left(90^{\circ}\right)$ and perpendicular to the capillary $\left(0^{\circ}\right)$. Evolution of polarization difference ratio with pump power (crossed circles).

\section{REFERENCES}

[1] D. V. Talapin, J.-S. Lee, M. V. Kovalenko, and E. V. Shevchenko, "Prospects of colloidal nanocrystals for electronic and optoelectronic applications," Chemical reviews, vol. 110, pp. 389-458, 2009.

[2] V. Klimov, A. Mikhailovsky, S. Xu, A. Malko, J. Hollingsworth, C. Leatherdale, H.-J. Eisler, and M. Bawendi, "Optical gain and stimulated emission in nanocrystal quantum dots," Science, vol. 290, pp. 314-317, 2000.

[3] J. Hu, L.-s. Li, W. Yang, L. Manna, L.-w. Wang, and A. P. Alivisatos, "Linearly polarized emission from colloidal semiconductor quantum rods," Science, vol. 292, pp. 2060-2063, 2001.

[4] D. V. Talapin, R. Koeppe, S. Götzinger, A. Kornowski, J. M. Lupton, A. L. Rogach, O. Benson, J. Feldmann, and H. Weller, "Highly emissive colloidal $\mathrm{CdSe} / \mathrm{CdS}$ heterostructures of mixed dimensionality," Nano letters, vol. 3, pp. 1677-1681, 2003.

[5] M. Zavelani-Rossi, M. G. Lupo, F. Tassone, L. Manna, and G. Lanzani, "Suppression of biexciton auger recombination in cdse/cds dot/rods: Role of the electronic structure in the carrier dynamics," Nano letters, vol. 10, pp. 3142-3150, 2010.

[6] C. Grivas, C. Li, P. Andreakou, P. Wang, M. Ding, G. Brambilla, L. Manna, and P. Lagoudakis, "Single-mode tunable laser emission in the single-exciton regime from colloidal nanocrystals," Nature communications, vol. 4, 2013.

[7] L. Carbone, C. Nobile, M. De Giorgi, F. D. Sala, G. Morello, P. Pompa, M. Hytch, E. Snoeck, A. Fiore, and I. R. Franchini, "Synthesis and micrometer-scale assembly of colloidal $\mathrm{CdSe} / \mathrm{CdS}$ nanorods prepared by a seeded growth approach," Nano letters, vol. 7, pp. 2942-2950, 2007.

[8] J. S. Kamal, R. Gomes, Z. Hens, M. Karvar, K. Neyts, S. Compernolle, and F. Vanhaecke, "Direct determination of absorption anisotropy in colloidal quantum rods," Physical Review B, vol. 85, p. 035126, 2012.

[9] J. L. Baker, A. Widmer-Cooper, M. F. Toney, P. L. Geissler, and A. P. Alivisatos, "Device-scale perpendicular alignment of colloidal nanorods," Nano letters, vol. 10, pp. 195-201, 2009.

[10]A. Rizzo, C. Nobile, M. Mazzeo, M. D. Giorgi, A. Fiore, L. Carbone, R. Cingolani, L. Manna, and G. Gigli, "Polarized light emitting diode by long-range nanorod self-assembling on a water surface," ACS Nano, vol. 3, pp. 1506-1512, 2009.

[11]Y. Gao et al, "Anisotropic Stimulated Emission from Aligned CdSe/CdS Dot-in-Rods," In Submission.

[12]Y. Huang, X. Duan, Q. Wei, and C. M. Lieber, "Directed assembly of one-dimensional nanostructures into functional networks," Science, vol. 291, pp. 630-633, 2001.

[13]J. Herrnsdorf, B. Guilhabert, Y. Chen, A. Kanibolotsky, A. Mackintosh, R. Pethrick, P. Skabara, E. Gu, N. Laurand, and M. Dawson, "Flexible blue-emitting encapsulated organic semiconductor DFB laser," Optics Express, vol. 18, pp. 25535-25545, 2010. 